\title{
A Study of Quality of Life and its Determinants among Hemodialysis Patients Using the KDQOL-SF Instrument in One Center in Saudi Arabia
}

\author{
Ahmed AL-Jumaih $^{* 1}$, Kamel Al-Onazi² ${ }^{2}$ Salih Binsalih², Fayez Hejaili ${ }^{3}$, Abdulla Al-Sayyari ${ }^{4}$ \\ (1) Medical Student, (2) Chairman, Department of Medicine, (3) Associate Professor of Medicine and (4) Professor of Medicine, King \\ Saud Bin Abdulaziz University for Health Sciences, Riyadh, Kingdom of Saudi Arabia
}

\begin{abstract}
Introduction: We aimed to assess quality of life (QOL) among Saudi hemodialysis (HD) patients and the impact on the QOL of a certain demographic and clinical factors.

Methods: The QOL was assessed using an Arabic version of Kidney Disease Quality of Life Instrument Short Form (KDQOL-SF). Mean scores were compared for individual domain scores and for the three composite summary scores, namely the mental component score (MCS), the physical component score (PCS) and kidneydisease component score (KDCS).

Results: The study included one hundred chronic HD patients from King Abdulaziz Medical City, Riyadh. The overall mean score was 60.4. Domains with very low scores were "cognitive function", "role-emotional", "role-physical" and "work status". Domains with high scores were "patient satisfaction", "dialysis staff encouragement" and "quality of social interaction". The mean scores for KDCS, MCS and PCS were 59.7, 54.2 and 52.7 respectively. KDC scores were higher among males and the married group. PCS scores were higher among males, patients aged $<40$ years, and the higher income group. MCS scores were higher among males and the higher income groups. There was a positive correlation between KDCS and $\operatorname{MCS}(\mathrm{r}=0.62, \mathrm{P}=0.0001)$; and between KDCS and PCS $(r=0.65, \mathrm{P}=0.0001)$.
\end{abstract}

Conclusion: We provided a detailed description of the QOL scores of a group of Saudi HD patients and the impact of certain factors on their QOL. Low scores were seen in the "work status", "cognitive function", "role-physical" and "role-emotional" while high scores were seen in "patient satisfaction", "dialysis staff encouragement" and "quality of social interaction" domains.

* Corresponding Author; Prof. Abdulla A Al-Sayyari, King Saud Bin Abdulaziz University for Health Sciences, PO Box 22490, Riyadh, 11426, KSA; E mail: aaalsayyari@gmail.com
Keywords: Hemodialysis; Kidney Disease; Quality of Life; Saudi Arabia

\section{The authors declared no conflict of interest}

\section{Introduction}

Patients' quality of life (QOL) assessment is gaining increasing importance in the medical literature [1]. Better QOL scores have been found to be associated with better compliance [2] and reduced morbidity and mortality [3, 4]. There are many tools used to assess QOL in patients. Some of these tools are specific for certain disease [5]. Kidney Disease Quality of Life Instrument Short Form (KDQOL-SF) has been used extensively for assessment ofQOL in kidney disease patients. It has been translated to many languages and validated in different ethnic groups [5]. A number of studies using KDQOL-SF to assess QOL in dialysis patients have recently been published [6-8]. In one study it was found that a positive responses to two questions in this tool ("Have you felt downhearted and blue?" and "Have you felt so down in the dumps that nothing could cheer you?"), were associated with increased risk of mortality and hospitalization [9]. No previous assessment of QOL in Saudi hemodialysis (HD) patients was performed. In this report we will describe the findings of the use of KDQOL-SF36 survey in Saudi patients in one HD center, compare them to other findings from other populations and find out if these findings are related to any socioeconomic or demographic characteristics in our patients.

\section{Methods}

The QOL was assessed using the KDQOL-SF36 instrument. The translated questionnaire's reliability was tested using methods of cross-cultural validations [5]. The survey contains 36 questions of which 15 are on demographic data and the rest cover 19 QOL domains. These 19 domains were grouped into three main domains. 
Table 1: The mean scores for each domain of the KDQOL-SF ${ }^{36}$ instrument among studied HD patients $(\mathrm{N}=100)$

\begin{tabular}{lll}
\hline & N & Mean (SD) \\
\hline PCS & $\mathbf{1 0 0}$ & $\mathbf{5 2 . 7}(\mathbf{2 3 . 4 )}$ \\
Physical Functioning & 100 & $56.4(29.1)$ \\
Role-Physical & 100 & $35.0(38.8)$ \\
Pain & 100 & $61.3(34.8)$ \\
General Health & 100 & $58.2(25.0)$ \\
MCS & $\mathbf{1 0 0}$ & $\mathbf{5 4 . 1}(\mathbf{2 4 . 5 )}$ \\
Energy/Fatigue & 100 & $56.5(28.9)$ \\
Social Function & 100 & $58.9(29.1)$ \\
Role Emotional & 100 & $37.5(44.6)$ \\
Emotional Well being & 100 & $63.7(26.8)$ \\
KDCS & $\mathbf{1 0 0}$ & $\mathbf{5 9 . 7}(\mathbf{1 5 . 8})$ \\
Symptoms/Problems List & 100 & $77.3(16.3)$ \\
Effect of Kidney Disease on daily life & 99 & $73.0(33.5)$ \\
Burden of Kidney Disease & 100 & $51.0(30.7)$ \\
Cognitive function & 100 & $25.6(9.5)$ \\
Work Status & 100 & $24.5(35.2)$ \\
Sexual Function & 24 & $81.2(23.3)$ \\
Quality of Social Interaction & 98 & $81.8(28.3)$ \\
Sleep & 100 & $66.8(24.4)$ \\
Social Support & 100 & $78.3(29.8)$ \\
Dialysis Staff Encouragement & 100 & $81.5(26.1)$ \\
Patient Satisfaction & 100 & $81.5(26.1)$ \\
Overall score & $\mathbf{1 0 0}$ & $\mathbf{6 0 . 4}(\mathbf{2 7 . 3 )}$ \\
\hline & & \\
& &
\end{tabular}

PCS: physical component summaries; MCS: mental component summaries; KDCS: kidney-disease component summaries

These are: a) Physical health components summary (PCS): physical functioning (10 items), role-physical (4 items), bodily pain (2 items), and general health (5 items); b) Mental health components summary (MCS): vitality/energy (4 items), social functioning ( 2 items), role emotional (5 items), and mental health (3 items) and c) Kidney disease component summary (KDCS): symptom / problem list (12 items), effects of kidney disease on daily life (8 items), burden of kidney disease (4 items), cognitive function (4 items), work status (2 items), sexual function ( 2 items), quality of social interaction (3 items), sleep (4 items), social support (2 items), dialysis staff encouragement ( 2 items) and patient satisfaction (1 item).

As per Hayes algorithm [10] the raw data obtained from the patients was first transformed to pre-coded numeric value of a $0-100$ possible range, with higher transformed scores always reflecting better quality of life. In the final step in the scoring process, items in the same scale were averaged together to create the scale score. Scores were also meaned according to summary scores: mental component summaries (MCS), physical component summaries (PCS) and kidney-disease component summaries (KDCS). One hundred randomly selected HD patients were enrolled between January and March 2009. They constituted $61.3 \%$ of chronic HD outpatients at King Abdulaziz Medical City, Riyadh. Thirty-three patients were randomly selected from each of the three dialysis shifts on alternate basis. The male patients were 68 and female patients 32. Demographic data was collected for all respondents as well as data on hospitalization, medication, dialysis adequacy, marital status, level of education, income, employment, emergency room (ER) visits, duration on dialysis, and cause of chronic kidney disease.

Data entry and verification was done initially by using an excel-spread sheet which was then exported to SPSS statistical package for windows (version 16). The reliability of the questionnaire was tested by Cronbach's Alpha. Chi-square test of significance was used to compare proportions for categorical variables. The t-test was used to compare means. Comparison of responses in different domains by different demographic characteristics was assessed by Fisher exact test for nonparametric variables and by 2 -tailed t-test for parametric variables.

\section{Results}

The total number of patients was 100 . Males represented $68.7 \%$ of the total. Their mean age was 53.4 years (SD 10.3 ) and $49 \%$ were aged 50 years or more. Their median and mean duration on dialysis was 5 years (SD 2.9). Sixty-four percent of patients were married. Forty-three percent of patients were retired, $28 \%$ were working full or part time, $38 \%$ were unemployed and $2 \%$ were studying (in schools/ colleges). Twenty-six percent of patients never went to school and only $4 \%$ attended a university.

Diabetic nephropathy was the most common single cause of renal failure among our patient population (37.3\%). The mean number of drugs taken was 8.9. The mean number for ER visits over the previous six months was 2.2. The mean number for hospital days during the previous 6 months was 8.9.

The mean score for each domain ranged from 24.5 for health status to 81.8 for quality of social interaction. The overall mean was 60.4 (SD 27.3). The scores for the kidney-disease component summary (KDCS), mental component summary (MCS) and the physical component summary (PCS) were 59.7 (SD 15.8), 54.2 (SD 24.5) and 52.7 (SD 23.4); respectively (Table-1). 
Table 2: The impact of various patient characteristics on scores in the three composite domains of the KDQOL-SF36 instrument among studied HD patients $(\mathrm{N}=100)$

\begin{tabular}{|c|c|c|c|c|c|c|}
\hline & \multicolumn{2}{|l|}{ KDC } & \multicolumn{2}{|l|}{ MCS } & \multicolumn{2}{|l|}{ PCS } \\
\hline & Score & P value & Score & P value & Score & P value \\
\hline \multicolumn{7}{|l|}{ Gender } \\
\hline Male & 64.4 & $\mathrm{P}=0.0001^{*}$ & 60.1 & $\mathrm{P}=0.001^{*}$ & 57.9 & $\mathrm{P}=0.001^{*}$ \\
\hline Female & 51.3 & & 42.5 & & 42.1 & \\
\hline \multicolumn{7}{|l|}{ Age } \\
\hline$<40$ years of age & 58.8 & $\mathrm{P}=0.45$ & 53.5 & $\mathrm{P}=0.7$ & 60.1 & $\mathrm{P}=0.02^{*}$ \\
\hline$>40$ years of age & 61.1 & & 55.2 & & 48.9 & \\
\hline \multicolumn{7}{|l|}{ Marital status } \\
\hline Married & 62.6 & $\mathrm{P}=0.03^{*}$ & 57.90 & $\mathrm{P}=0.05$ & 52.40 & $\mathrm{P}=0.7$ \\
\hline Unmarried & 56 & & 48.5 & & 54 & \\
\hline \multicolumn{7}{|l|}{ Educational level } \\
\hline Below secondary school & 61 & $\mathrm{P}=0.6$ & 55.5 & $\mathrm{P}=0.7$ & 50 & $\mathrm{P}=0.7$ \\
\hline Above secondary School & 59.2 & & 53.5 & & 56.7 & \\
\hline \multicolumn{7}{|l|}{ Cause of Renal Failure } \\
\hline Diabetic & 60.3 & $\mathrm{P}=0.9$ & 57.8 & $\mathrm{P}=0.3$ & 50.7 & $\mathrm{P}=0.4$ \\
\hline Non-Diabetic & 60.2 & & 52.3 & & 54.6 & \\
\hline \multicolumn{7}{|l|}{ Duration on Dialysis } \\
\hline$<4$ years & 60.8 & $\mathrm{P}=0.8$ & 56.1 & $\mathrm{P}=0.6$ & 56.1 & $\mathrm{P}=0.7$ \\
\hline$>4$ years & 60.2 & & 53.6 & & 53.6 & \\
\hline \multicolumn{7}{|l|}{ Income } \\
\hline$<4000 \mathrm{SR} / \mathrm{month}$ & 58.1 & $\mathrm{P}=0.1$ & 51.2 & $\mathrm{P}=0.03^{*}$ & 51.3 & $\mathrm{P}=0.01^{*}$ \\
\hline$>4000 \mathrm{SR} / \mathrm{month}$ & 66.5 & & 63.0 & & 59.6 & \\
\hline
\end{tabular}

PCS: physical component summaries; MCS: mental component summaries; KDCS: kidney-disease component summaries.

* Statistically significant

The effects of gender, age, marital status, education level, cause of renal failure, duration of dialysis and income on the three major composite scores are shown (Table-2). KDC, MCS and PCS scores were all significantly higher in males compared to females. PCS scores were significantly higher among patients aged $<40$ years. KDC scores were significantly higher among the married. MCS and PCS scores were significantly higher among the higher income group. Scores were not significantly affected by level of education, duration on dialysis or cause of renal failure.

We also found a positive correlation between the scores for KDCS and MCS ( $\mathrm{r}=0.62, \mathrm{P}=0.0001)$ as well as between KDCS and PCS $(r=0.65, P=0.0001)$ (Figures 1-2)

Table- 3 shows the scores in the KDCS, MCS and PCS domains of the KDQOL-SF ${ }^{36}$ instrument in this study compared to results from six other countries from North and South America, Middle East, Far East and Europe. The score for KDCS among Saudi patients in this study is the lowest among all seven countries. As for MCS and PCS domains, the sores among Saudi patients in this study are higher than scores reported from Europe, Japan, USA and Korea but lower than scores reported from Brazil and Turkey.

\section{Discussion}

Previous experiences with the Arabic version of the SF-36 instrument have proved it to be valid and robust [13, 14]. In one study using this tool in a cohort of normal Saudis, it was found that Saudis have significantly higher vitality scores, but significantly lower physical functioning, social functioning, and general health perception scores than the general US population [15]. In a study in Lebanon, using an Arabic version of SF-36, rural residents had higher vitality scores than urban residents and the QOL score of men was higher than that of women [13].

Monitoring a patient's physical and mental status and his/her subjective status of well- being, together known as QOL measurements, is of particular importance in 
Figure 1: Scatterplot demonstrating positive correlation between KDCS and MCS scores of the KDQOL-SF36 instrument among studied HD patients $(r=0.62, P=0.0001)$

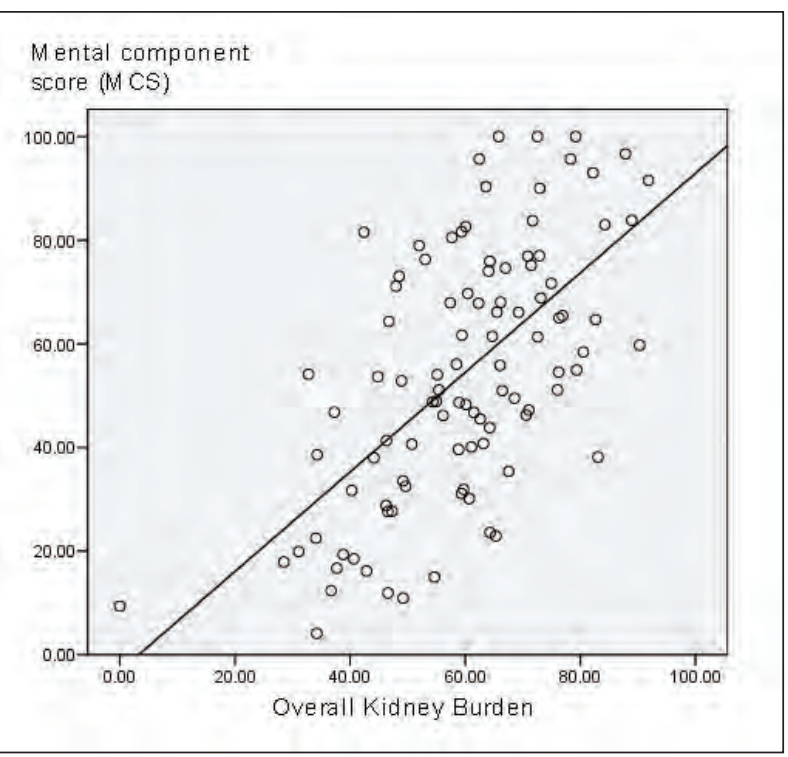

patients with end stage renal disease (ESRD). Among ESRD patients, better quality of life is associated with better compliance with therapy and improved survival. Five-point higher scores for the QOL dimensions of physical health, mental health and kidney disease targeted issues were associated with a $4-8 \%$ reduction in the risk of hospitalization, and a $9-23 \%$ reduction in mortality risk [3].

The current study provides a detailed description of the QOL scores of a group of Saudi HD patients and the impact of certain factors on their QOL.

In this study, the mean overall score in the 19 domains was 60.4 (range 24.5-81.4). On comparing the scores in the three composite domains in this cohort of patients, we found that the lowest score (52.7) was seen in PCS domain followed by MCS domain (54.2), while KDSC domain had the highest score (59.7). Low scores $(<50)$ were seen in the "work status", "cognitive function", "role-physical" and "role-emotional" (scores 24.5, 25.6, 35 and 37.5 respectively). This can be attributed to the fact that most of the patients studied had chronic comorbidities and a lot of them were unemployed. High scores $(>80)$ were seen in "patient satisfaction", "dialysis staff encouragement" and "quality of social interaction" domains.

MCS and PCS scores were significantly higher among the higher income group. This is probably because they had good financial support which helped them in coping with life difficulties and decrease life stresses. Previous studies have also shown that unemployment and lower income
Figure 2: Scatterplot demonstrating positive correlation between KDCS and PCS scores of the KDQOL-SF36 instrument among studied $\mathrm{HD}$ patients $(\mathrm{r}=0.65, \mathrm{P}=0.0001)$

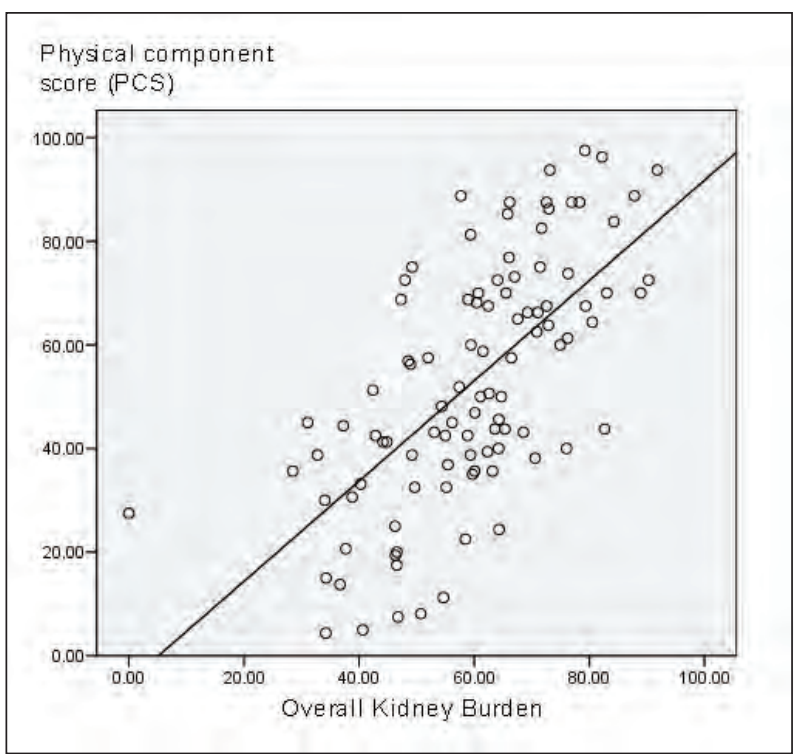

level were independently and significantly associated with lower scores of PCS and/or MCS in several generic and kidney disease-targeted scales [16-18]. In the DOPPS study, on the other hand, no differences were seen due to income, marital status or educational level [4]. In this study, males scored higher than females in the three composite domains, which might be explained in part by the fact that more males than females in this sample were married and earned good income. Similar results were reported from USA, with worse scores being seen in females [19]. A study in UK using the same instrument, however, showed lower scores in male patients [20]. In a report on longitudinal assessment of QOL in peritoneal dialysis patients in the UK, QOL was observed to decline over time. Worse overall QOL dimension scores (physical health, mental health, kidney disease issues and patient satisfaction) were more likely to be seen in male and Asian patients in the UK [20].

Compared to patients from different countries, this sample of Saudi HD patients had lower scores in the "roleemotional", "role-physical" and "cognitive function" domains [4, 8-10]. On the other hand, our patients scored higher in the "quality of social interaction", "patient satisfaction" and "dialysis staff encouragement" domains. These findings can be explained by the fact that in our society we have strong social support, family bonds, well-trained staff and well established dialysis centers. In a report from another Arab Moslem country, Egypt, HD patients had much lower MCS (38.8 versus54.2) and PCS (34 versus 52.7) scores than our Saudi patients [21]. 
Table 3: Comparison between scores in the KDCS, MCS and PCS domains of the KDQOL-SF36 instrument in the current study and six different countries

\begin{tabular}{llllllll}
\hline Country & KSA $^{*}$ & Europe [3] & Japan [11] & USA [4] & Korea [10] & Brazil [8] & Turkey [12] \\
\hline PCS & 52.7 & 35.5 & 41.8 & 33.1 & 53 & 60 & 62 \\
MCS & 54.1 & 43.3 & 44.8 & 46.6 & 51 & 68 & 71 \\
KDCS & 59.7 & 69.9 & 75.8 & 71.1 & 62.9 & 67.9 & 63.8 \\
\hline
\end{tabular}

PCS: physical component summaries; MCS: mental component summaries; KDCS: kidney-disease component summaries.

* Current study

Our patients scored high in sexual function. However this could be a misleading result and may not reflect the true status as this question was answered by only $24 \%$ of patients. The others were too embarrassed to respond to this question and this may have resulted in bias.

\section{Conclusions}

The current study provides a detailed description of the QOL scores of a group of Saudi HD patients and the impact of certain factors on their QOL. Low scores were seen in the "work status", "cognitive function", "role-physical" and "role-emotional" while high scores were seen in "patient satisfaction", "dialysis staff encouragement" and "quality of social interaction" domains. KDC scores were higher among males and the married group. PCS scores were higher among males, patients aged $<40$ years and the higher income group. MCS scores were higher among males and the higher income groups.

\section{References}

1. Valderrábano F, Jofre R, López-Gómez JM. Quality of life in end-stage renal disease patients. Am J Kidney Dis. 2001 Sep;38(3):443-64.

2. DeOreo PB. Hemodialysis patient-assessed functional health status predicts continued survival, hospitalization, and dialysis-attendance compliance. Am J Kidney Dis. 1997 Aug;30(2):204-12.

3. Mapes DL, Lopes AA, Satayathum S, McCullough KP, Goodkin DA, Locatelli F, Fukuhara S, Young EW, Kurokawa K, Saito A, Bommer J, Wolfe RA, Held PJ, Port FK. Health-related quality of life as a predictor of mortality and hospitalization: the Dialysis Outcomes and Practice Patterns Study (DOPPS). Kidney Int. 2003 Jul;64(1):339-49.

4. Lopes AA, Bragg-Gresham JL, Satayathum S, McCullough K, Pifer T, Goodkin DA, Mapes DL, Young EW, Wolfe RA, Held PJ, Port FK; Worldwide Dialysis Outcomes and Practice Patterns Study Committee.
Health-related quality of life and associated outcomes among hemodialysis patients of different ethnicities in the United States: the Dialysis Outcomes and Practice Patterns Study (DOPPS). Am J Kidney Dis. 2003 Mar;41(3):605-15.

5. WareJEJr, SherbourneCD. TheMOS36-item short-form health survey (SF-36). I. Conceptual framework and item selection. Med Care. 1992 Jun;30(6):473-83.

6. Ibrahim S, El Salamony O. Depression, quality of life and malnutrition-inflammation scores in hemodialysis patients. Am J Nephrol. 2008;28(5):784-91.

7. Hays RD, Kallich JD, Mapes DL, Coons SJ, Carter WB. Development of the kidney disease quality of life (KDQOL) instrument. Qual Life Res. 1994 Oct;3(5):32938.

8. Duarte PS, Ciconelli RM, Sesso R. Cultural adaptation and validation of the "Kidney Disease and Quality of Life--Short Form (KDQOL-SF 1.3)" in Brazil. Braz J Med Biol Res. 2005 Feb;38(2):261-70.

9. Lopes AA, Bragg J, Young E, Goodkin D, Mapes D, Combe C, Piera L, Held P, Gillespie B, Port FK; Dialysis Outcomes and Practice Patterns Study (DOPPS). Depression as a predictor of mortality and hospitalization among hemodialysis patients in the United States and Europe. Kidney Int. 2002 Jul;62(1):199-207.

10.Park HJ, Kim S, Yong JS, Han SS, Yang DH, Meguro M, Han CW, Kohzuki M. Reliability and validity of the Korean version of Kidney Disease Quality of Life instrument (KDQOL-SF). Tohoku J Exp Med. 2007 Apr;211(4):321-9.

11. Fujisawa M, Ichikawa Y, Yoshiya K, Isotani S, Higuchi A, Nagano S, Arakawa S, Hamami G, Matsumoto O, Kamidono S. Assessment of health-related quality of life in renal transplant and hemodialysis patients using the SF-36 health survey. Urology. 2000 Aug 1;56(2):201-6.

12. Yildirim A, Ogutmen B, Bektas G, Isci E, Mete M, Tolgay HI. Translation, cultural adaptation, initial reliability, and validation of the Kidney Disease and 
Quality of Life-Short Form (KDQOL-SF 1.3) in Turkey. Transplant Proc. 2007 Jan-Feb;39(1):51-4.

13. Sabbah I, Drouby N, Sabbah S, Retel-Rude N, Mercier M. Quality of life in rural and urban populations in Lebanon using SF-36 health survey. Health Qual Life Outcomes. 2003 Aug 6;1:30.

14. Khoudri I, Ali Zeggwagh A, Abidi K, Madani N, Abouqal R. Measurement properties of the short form 36 and health-related quality of life after intensive care in Morocco. Acta Anaesthesiol Scand. 2007 Feb;51(2):18997.

15. Coons SJ, Alabdulmohsin SA, Draugalis JR, Hays RD. Reliability of an Arabic version of the RAND-36 Health Survey and its equivalence to the US-English version. Med Care. 1998 Mar;36(3):428-32.

16. Rasgon S, James-Rogers A, Chemleski B, Ledezma M, Mercado L, Besario M, Trivedi J, Miller M, Dee L, Pryor L, Yeoh H. Maintenance of employment on dialysis. Adv Ren Replace Ther. 1997 Apr;4(2):152-9.
17. Roscoe JM, Smith LF, Williams EA, Stein M, Morton AR, Balfe JW, Arbus GS. Medical and social outcome in adolescents with end-stage renal failure. Kidney Int. 1991 Nov;40(5):948-53.

18. van Manen JG, Korevaar JC, Dekker FW, Reuselaars MC, Boeschoten EW, Krediet RT; NECOSAD Study Group. Netherlands Cooperative Study on Adequacy of Dialysis. Changes in employment status in end-stage renal disease patients during their first year of dialysis. Perit Dial Int. 2001 Nov-Dec;21(6):595-601.

19. Sehgal AR. Outcomes of renal replacement therapy among blacks and women. Am J Kidney Dis. 2000 Apr;35(4 Suppl 1):S148-52.

20. Bakewell AB, Higgins RM, Edmunds ME. Quality of life in peritoneal dialysis patients: decline over time and association with clinical outcomes. Kidney Int. 2002 Jan;61(1):239-48.

21. Assal HS, Emam HM, Abdul Ghaffar N Healthrelated Quality of Life among Egyptian Patients on Hemodialysis J Med Sci 2006 6:314-320. 